\title{
Etre présent en ligne : culture et structure des réseaux sociaux d'Internet
}

\section{Comment construit-on concrètement sa présence en ligne ? Au travers d'une analyse théorique et d'une expérience conduite sur Facebook, le sociologue Antonio Casilli montre comment la structure des communautés sur Internet et les pratiques de leurs membres évoluent conjointement.}

Chaque jour, nous avons recours à des descriptions,

Antonio A. Casilli, maitre de conférences à Télécom ParisTech et chercheur associé à l'École des hautes études en sciences sociales $^{1}$

1 Le présent texte est une version remaniée et augmentée des analyses initialement proposées dans les chapitres « Ceci est mon corps (en ligne) ", «Un profil comme le mien » et « Mon réseau est une image de moi » de mon ouvrage, Les Liaisons numériques. Vers une nouvelle sociabilité? paru au Seuil en $2010[\mathrm{NdA}]$.

2 Les numéros entre crochets renvoient à la bibliographie en fin d'article.

à des images ou à des symboles censés représenter notre présence en ligne. Les communications assistées par ordinateur grouillent de traces de l'identité de leurs utilisateurs. À peine suggérés (un nom, la photo d'un visage) ou développés d'une main experte (les créatures 3D des jeux vidéo), ces indices d'activité humaine font la spécificité et le succès des médias sociaux contemporains. «Personnaliser son profil », ouvrir des fenêtres de « dialogue », interagir avec une « interface » : les métaphores que nous empruntons pour décrire nos usages inscrivent l'expérience numérique dans la sphère intime, la partie immanente de nos existences. Elles sont autant de manifestations de notre manière de penser ces technologies, de les associer au domaine de notre corporéité.

La présence en ligne passe donc par une forme de monstration - naturaliste ou idéalisée - du corps de l'usager. En mettant en scène son corps, un usager arrive à être «téléprésent » dans un échange en ligne et peut interagir avec les autres par l'entremise d'un personnage qui est son représentant à l'écran. Couramment, on appelle ce personnage «avatar». Ce terme sanskrit, qui désignait dans la tradition hindoue l'incarnation d'une divinité, renvoie aujourd'hui à une photo ou à un personnage numérique dans des services aussi variés que Twitter ou des univers multijoueurs [1] ${ }^{\mathbf{2}}$. Une incarnation faite de pixels, si l'on veut, derrière laquelle un acteur social cherche à exprimer ses exigences, ses goûts, ses dispositions.
Une typologie très accessible et efficace de traces corporelles en ligne consiste à les classer par leur format. Nous pouvons alors en distinguer trois types différents : mono, bi et tridimensionnelles.

\section{«Vrais » noms et pseudonymes: enjeux de la dénomination en ligne}

Les descriptions corporelles monodimensionnelles s'étendent sur une ligne à peine : un identifiant à l'écran ou une adresse électronique peuvent suffire. Tout comme les noms dans la vie quotidienne, ils représentent des critères élémentaires qui nous aident à nous faire une idée des caractéristiques des personnes que nous rencontrons pour la première fois. Dans la réalité quotidienne, nous nous servons des noms pour trier, deviner, anticiper les caractéristiques de nos interlocuteurs. «Qu'y a-t-il dans un nom? » : sans être Shakespeare, nous nous posons cette question chaque jour, dans nos rencontres en face-à-face tout comme lors de nos flâneries en ligne. Leur sexe, par exemple, ou leur origine, et parfois leur âge. Un «艾未未» s'affichant sur Sina Weibo se présentera à notre esprit d'une manière complètement différente du «bruce. kirkpatrick@nyu.edu »qui fait son apparition dans notre boîte mail. Ces noms induisent d'emblée dans notre esprit des jeux de caractéristiques propres à ces usagers, les styles, vocabulaires, gestuelle des personnes qui s'en servent.

La différence cependant réside dans le fait que les noms à l'écran ont été choisis et non imposés, 
comme les identités civiles. C'est pourquoi hors ligne, en général, les noms réels nous réservent moins de surprises. Comme nous le rappelle Baptiste Coulmont [2], si les noms ou les prénoms de l'état civil révèlent autant de contraintes sociales (lois de l'État, équilibres familiaux, assimilation culturelle) que d'aspirations individuelles à exprimer un « soi » profond, les traces monodimensionnelles sur Internet insistent principalement sur ce deuxième aspect. Elles se veulent ainsi des modes de dénomi- d'animal ou de personnage célèbre, les internautes veulent suggérer qu'ils partagent quelques qualités avec ces derniers [3].

Une catégorie à part de descriptions monodimensionnelles est représentée par les formules décrivant des actions, des expressions et des gestuelles parfois extrêmement complexes. La forme la plus répandue est sans aucun doute celle des émoticônes, ou smileys. De l'omniprésent sourire : -) aux variations classiques : clin d'œil ; -), pleurer :'- (,

\section{“ Une incarnation faite de pixels derrière laquelle un acteur social cherche à exprimer ses exigences, ses goûts, ses dispositions}

nation autonome, des prismes à travers lesquels la volonté de présence en ligne des usagers peut être étudiée.

Bien sûr, la déception rôde. Si, hors ligne, l'onomastique est un outil d'attestation officielle de l'identité, réglementé par les pouvoirs étatiques afin de limiter les malentendus et les abus, au sein des communautés d'Internet, les traces monodimensionnelles sont «liquides » et fugaces. Certes, les béhémots du Web, tel Google, cherchent depuis longtemps à mettre en place des politiques de «noms réels » (real names) sur leurs services. Mais pour chaque identifiant unique qui est imposé sur les plateformes commerciales, tout usager accumule une pléthore de pseudonymes (et dans certains cas, d'identités anonymes...) qui compensent ces tentatives d'immatriculation et de traçage. Pareillement, les faux noms (fakes) relèvent moins de l'usurpation d'identité que de la quête d'autonomie de leurs usagers.

Qu'elles correspondent ou non à l'identité civile de leurs utilisateurs, ces traces de présence illustrent des traits et des références culturelles des personnes qui y ont recours. Quoique non nécessairement cohérentes avec l'apparence réelle de leurs utilisateurs (mais quelle représentation peut espérer l'être?), elles restent d'un certain point de vue toujours authentiques. En choisissant un nom crier : -O, etc. Depuis sa première occurrence documentée en 1982, cette trace monodimensionnelle ajoute une dimension informelle ou emphatique aux messages. L'imagination des internautes a bientôt entraîné une grande variété de conventions graphiques. Certaines expriment des gestes et des attitudes complexes, comme dans les émoticônes de style manga, très utilisées en Asie : $\backslash\left(\wedge^{\mathrm{O}^{\wedge}}\right) /(\ll$ lever les bras en signe de jubilation », les parenthèses délimitant le visage, les circonflexes figurant les yeux et le «o » la bouche), $\left(>_{-}<\right)$(« être sur le point d'exploser de rage $»),\left({ }^{\wedge}{ }_{-}^{\wedge}{ }^{\prime}\right)($ («transpirer $»$, l'apostrophe représentant une goutte de sueur), etc.

\section{Photos et présentation de soi}

Quand les descriptions transmises en réseau s'étendent sur plusieurs lignes de texte ou de pixels, elles sont des traces bidimensionnelles. Elles peuvent cependant être écrites (profils, narrations, phrases) ou audiovisuelles (photos, images en mouvement). Les fiches personnelles d'un site de rencontres, par exemple, fournissent de brèves présentations de l'apparence et du style de chaque membre. C'est également le cas des blogueurs qui choisissent un petit portrait photo ou un dessin humoristique associé aux articles publiés sur leur site et aux commentaires sur ceux d'autrui. 
Quoi qu'il en soit, les internautes ne cessent d'impliquer leur corps dans l'échange, capturant ainsi leur expressivité, leur sensibilité, leurs réactions aux contenus partagés. Les sites de stockage de photos ou de vidéos comme Photobucket ou Dailymotion en sont de bons exemples. Par le biais de ces traces corporelles, l'internaute se met en scène dans son quotidien. Il s'agit ainsi non seulement d'une manière de raconter sa vie et d'intéresser les autres, mais aussi de gérer l'image qu'il ou elle donne de soi.

Mieux vaut insister encore sur ce point: que les photos ou les vidéos mises en ligne soient réalistes ou fantaisistes, peu importe, pourvu qu' elles expriment une avant l'arrivée d'Internet, la sociologie interactionniste avait analysé les comportements en société comme des processus dialectiques, des allers et retours incessants entre une scène (espace de la monstration de soi) et des coulisses (lieux en principe inaccessibles au public, où l'acteur peut ne pas être ce qu'il dit être quand il est sur la scène). Si l'on remplace les notions de «scène» et de «coulisses » par celles de « réseau » et de « vie hors ligne », le cadre théorique tient toujours.

Selon Julia Velkovska et Valérie Beaudouin, «les différentes composantes del' espace communicationnel [...] peuvent être interprétées comme constituant un cadre de participation, au sens de Goffman, c' est-à-dire

\section{"6 Autant d'efforts seraient incompréhensibles si ces avatars se limitaient à être de simples portraits 3D de leurs créateurs "9}

présence. D'ailleurs, les portraits d'utilisateurs affichant leur visage de manière reconnaissable ne sont potentiellement pas plus « vrais » qu'une photo retouchée qui les embellit ou les déguise. Les techniques de correction ou d'amélioration de l'image photographique, pour l'historien André Gunthert, ont longtemps été considérées comme des tabous. Mais «depuis la vulgarisation des logiciels de traitement d'image, n'importe quel amateur peut aujourd'hui se confronter à l'expérience déroutante, non de la disparition du photographique, mais de la manipulation de sa substance même » [4].

Le sujet représenté pose, joue avec la lumière et le cadrage pour donner une certaine impression physique. Il n'y a déjà à ce stade plus rien de vrai et de naturel : le même gros plan d'un visage peut transmettre chaleur et participation (ou au contraire froideur et distance) selon qu' on y ajoute un filtre de couleur jaune ou bleue, qu' on élargit ou resserre le cadre. Les internautes apprennent à gérer ces codes de mise en scène du corps et, comme dans la vie hors ligne, ils apprennent vite à adapter leur comportement et leurs gestes selon l'impression qu'ils cherchent à donner à leurs interlocuteurs.

Il y a une très forte continuité entre les pratiques de gestion des traces corporelles sur les réseaux socioinformatiques et la «présentation de soi » étudiée par Erving Goffman dans son ouvrage de 1959 [5]. Bien comme délimitant l'accès des participants à un territoire commun et aux événements qui s'y produisent » [6]. La représentation à l'écran d'un utilisateur ne doit alors pas être envisagée comme un outil d'identification, nous permettant ou non de le reconnaître si on le croise dans un espace du quotidien hors ligne, mais avant tout comme un outil de positionnement et de recherche de reconnaissance au sein de la communauté en ligne où il est employé.

La question de la vérité du corps mis en présence sur Internet s'éloigne et devient moins significative. On peut en voir une démonstration parfaite dans les images autoproduites que les usagers partagent sur les blogs et les forums de discussion consacrés aux troubles des conduites alimentaires. Constamment stigmatisés dans les médias qui les qualifient de « pro-ana », d'apologètes de l'anorexie, ces services fournissent surtout un soutien et un sens de proximité émotionnelle à des jeunes gens en détresse [7]. Pour ce faire, et pour trier entre personnes souffrant de troubles du comportement alimentaire (TCA) et simples flâneurs animés par une curiosité morbide, ils doivent constamment demander des «preuves d'identité ». C'est à travers des traces bidimensionnelles (récits d'anorexiques, photos certifiant des «progrès » dans le trouble ou dans la guérison, témoignages vidéo) que les intéressés 
attestent leur droit d'appartenance à la communauté. Ils envoient ces gages à leurs correspondants en ligne. À les regarder, on est saisi par la sensation d'avoir affaire à des images dont tous les détails sont authentiques, mais dont l'ensemble est néanmoins fictif. Tout est extrêmement construit : face à un miroir ou à une webcam, les postures sont minutieusement soignées, les gestes codifiés à l'extrême, le cadrage fonctionnel à la valorisation de certaines parties du corps (bras, hanches, côtes). Entre photo-retouches et montages, la mise en scène est souvent tellement extravagante qu' elle finit par rendre méconnaissable la prétendue « vérité du corps » qu' elle était censée certifier.

\section{Lavatar, ou le corps en puissance}

Pour présenter le troisième type de traces corporelles, nous citerons les avatars tridimensionnels. Les personnages interactifs que l'on personnalise et que l'on fait évoluer dans les «MMORPG » (acronyme en anglais des « jeux en ligne massivement multijoueurs ») ou dans les univers virtuels en ligne sont peut-être les représentations les plus spectaculaires du corps à l'écran. Mais elles ne sont en réalité pas les plus répandues. Aujourd'hui, seulement un dixième des utilisateurs d'Internet s'en seraient en effet servi. Même si les estimations les plus optimistes annoncent que leur population pourrait atteindre un milliard d' « individus virtuels » en 2017, le pourcentage des effectifs (c'est-àdire des utilisateurs actionnant un ou plusieurs avatars 3D) ne dépasserait pas $22 \%$ des internautes haut débit à cette date [8].

Et pour cause: construire un avatar demande un savoir-faire technique et un apport financier parfois importants. Il faut s'abonner à des services en ligne deux semaines de travail à temps plein - ce qui semble restreindre considérablement le nombre d'utilisateurs.

Autant d'efforts seraient incompréhensibles si ces avatars se limitaient à être de simples portraits 3D de leurs créateurs. Ils représentent en réalité beaucoup plus que cela : ce sont les figures de ce que leur corps est en puissance, des alter ego qui incarnent leurs rêves de pouvoir, de beauté et de bonheur. Ce n'est pas un hasard si Alter Ego est aussi le titre choisi par le photographe anglais Robbie Cooper pour son ouvrage de 2007 sur les membres de mondes multijoueurs en ligne [9]. Ce livre de témoignages répertorie les imaginaires du corps au sein des cultures numériques en présentant soixante fiches d'utilisateurs de services en ligne contenant leur photographie, une capture d'écran de leur personnage et un petit entretien. Pour certains, l'avatar est un double de l'image photo-réaliste de leur corps hors ligne. Ils ont la même apparence, la même morphologie, les mêmes contenances. D'autres s'en servent pour figurer le corps qu'ils voudraient avoir. Un jeune homme frêle se représente ainsi comme un démon à la peau bleue et aux épaules massives - un power avatar, personnage monstrueux exprimant l'envie d'endurance et de puissance physique de son créateur. Un adolescent obèse se voit comme un homme mûr de constitution moyenne. Son personnage en ligne semble révéler une aspiration d'amélioration de soi, une potentialité du devenir de son corps : «Plus tard, je veux ressembler à ça. » Mais, dans le livre de Robbie Cooper, on trouve aussi le cas d'une jeune mère, son nouveau-né dans les bras et la silhouette marquée par son récent accouchement, qui s' imagine en odalisque sensuelle. Il s' agit là de la nostalgie d' une apparence perdue et en même temps de la volonté affirmée de revenir à son idéal physique.

\section{“6 Toute trace 3D met en scène des corps «en puissance »}

ou acheter des logiciels de graphisme 3D qui, tout en étant de plus en plus répandus, ne sont pas accessibles à tout le monde. C'est aussi une question d'emploi du temps. Créer un personnage virtuel prend quelques minutes, mais pour le «customiser » convenablement et lui donner l'apparence souhaitée, il faut compter en moyenne soixante-dix heures : c'est l'équivalent de
Que l'image que l'internaute a de son corps se projette dans le futur, se fige dans le passé ou idéalise le présent, toute trace 3D (même si elle ressemble fidèlement à celle de son créateur) met en scène des corps « en puissance ». En exprimant les desiderata liés à sa présence physique, l'internaute forme un projet de soi qui se fait, dans les réseaux, projet de corps. 


\section{Technologies du soi, technologies du nous}

Qu'elles soient monodimensionnelles, bidimensionnelles ou tridimensionnelles, ces représentations numériques sont des repères cognitifs qui nous aident à nous faire une idée des caractéristiques physiques de nos interlocuteurs en ligne. Elles fournissent, sinon une description fiable de l'apparence des personnes qu'elles représentent, du moins des indications parfois précises sur leur sensibilité et leur comportement. La question de la présence en réseau s'articule alors avec la construction d'une identité corporelle éminemment déclarative et performative : «[Sur Internet] peut-être plus qu'ailleurs, souligne Marie Bergström, le soi est nommé, mis à l'écrit et donc en récit. L'identité numérique comporte ainsi un caractère performatif dans la mesure où nous devons supposer que l'interlocuteur est ce qu'il revendique être [10]. »

«Customiser », adapter les images, projeter des aspirations : la pratique des réseaux sociaux en ligne atteste du «souci de soi » de centaines de millions d'internautes engagés, à un moment historique précis, dans des activités de négociation d'une "présence corporelle assistée par ordinateur ». Michel Foucault aurait parlé de « technologie du soi » [11] : à travers la projection des traces corporelles se met en place un procédé réalisant dans le corps même le travail de réflexion sur soi, de déchiffrement des désirs et des aspirations personnelles. Le caractère déclaratif et performatif sert finalement à harmoniser avec un milieu social choisi. La transformation que l'usager cherche à opérer sur lui-même rejoint la transformation plus ample de la communauté composée par les personnes avec lesquelles il ou elle interagit.

Il y a donc une dimension collective de la construction de la présence en ligne. Mais quels sont les outils théoriques et empiriques les plus adaptés pour les décrire et pour en saisir le fonctionnement? Les recherches de la décennie passée - portant sur les premières manifestations du Web social tels les blogs ou les pages personnelles - ont privilégié des approches microsociologiques. Tantôt appréhendées comme des signes de la prévalence d'un « individualisme expressif » [12], tantôt comme des «techniques relationnelles» s'inscrivant dans des dynamiques intersubjectives [13]. Mais les grandes plateformes sociales des dernières années - Twitter, Youtube et Facebook en tête - imposent d'autres approches, plus attentives aux dynamiques de négociation collective de la présence. Le questionnement a donc évolué, en passant du micro ( quelles sont les motivations des individus qui adoptent une certaine modalité de présence? ») au macro («quelles sont les structures des groupes qui permettent une certaine modalité de présence?»). Les outils empiriques se sont renouvelés conformément. De plus en plus d'études adoptent des approches expérimentales et s'appuient sur l'analyse structurale des réseaux sociaux pour illustrer les patterns d'adaptation de la présence sur les médias sociaux [14, 15].

\section{Une expérience sur Facebook}

À ce jour, l'étude des manières dont la présence en ligne des utilisateurs des médias sociaux se forme est notamment menée par le biais de méthodes statistiques. La décennie actuelle est en effet dévolue aux big data, c' est-à-dire à la collecte de larges bases de données sur les profils et les réseaux personnels en ligne. Ces pratiques ne vont pas sans soulever de nombreux problèmes éthiques et de protection de la vie privée [16]. Afin de minimiser ces difficultés, nous proposons une approche de la présence en ligne et de la formation de réseaux sociaux sur un média social généraliste basée sur une étude empirique conduite à petite échelle, limitant ainsi l'intrusion dans la sphère privée des enquêtés.

Une mini-expérience menée sur Facebook se prête à illustrer les divers avantages de cette démarche. Pendant une période de cinquante jours, nous avons observé comment diverses modalités de présence dans un profil d'utilisateur finissent par influencer de manière différente les structures sociales reliées à ce profil même ${ }^{3}$. Quoique très simple, cette expérience permet de se familiariser avec les principaux concepts de la sociologie des réseaux.

À cause de son caractère de médium généraliste, Facebook a été choisi en tant que terrain pour notre expérience. Depuis sa création en 2004 comme « trombinoscope virtuel » pour les étudiants de l'université de Harvard, la plateforme a subi maints remaniements. Au fil des années, le service s'est d'abord ouvert à tous les universitaires étasuniens, puis a progressivement accueilli des utilisateurs de tous âges, nationalités et niveaux de scolarité. Pratiquement chaque semaine, de nouvelles fonctionnalités étaient ajoutées : des manières innovantes d'agréger et de présenter les contenus mis en ligne par les utili- 
sateurs, des codes d'interaction mieux adaptés à un public de plus en plus hétérogène et - de façon plus controversée - des clauses d'utilisation de moins en moins respectueuses de la vie privée. Mais ce qui n'a jamais changé est sa structure, régie par un algorithme appelé EdgeRank, qui classe et interconnecte des profils : chaque utilisateur a une fiche personnelle contenant une adresse électronique, une photo, une liste d'amis et une courte description de ses goûts et activités. Bien sûr, qui dit «ami » dans ce contexte ne dit pas forcément «membre d'un groupe de camarades » [17, 18]. L'algorithme de Facebook permet de connecter le profil de chacun de ses membres à celui de personnes proches - mais aussi de collègues, de vieilles connaissances perdues de vue - et, par leur intermédiaire, de former de nouveaux liens avec des inconnus. Dans ce contexte, un «ami » n'est qu' un usager auquel on accorde l'accès à des contenus spéciaux (textes, liens internet, musiques, d'améliorer sa position structurale : il aura accès à davantage d'informations, il sera plus influent, ses actions auront un poids plus important [21].

La petite expérience entreprise sur Facebook cherchera alors à répondre à la question suivante: «Est-ce qu'en améliorant sa présence via son profil, un utilisateur arrive à maximiser son capital social ? »

\section{Un carnet d'observations en ligne}

Ce qui suit est un extrait du carnet d'observations mis en place à cette occasion.

Lundi 27 avril 2009. Je viens de créer à mon nom deux profils d'utilisateurs sur Facebook. Le profil $1 \mathrm{~s}$ 'appelle pour l'instant Tony Cas. Le profil 2, Ant Cas. Pourquoi deux? Parce que, comme dans les expériences de laboratoire, il faut pouvoir comparer deux entités initialement identiques, dont l'une sera soumise à une stimulation positive, l'autre invariable dans ses condi-

\section{'c Est-ce qu'en améliorant sa présence via son profil, un utilisateur arrive à maximiser son capital social?}

vidéos) et à des renseignements réservés. Cet accès privilégié à des informations confidentielles, généralement défendu au tout-venant des usagers, représente la vraie valeur ajoutée d'une bonne liste d'amis sur Facebook.

Si la notion d' «amitié en ligne » ne doit donc pas être interprétée comme une simple transposition de l'amitié en chair et en os, ce que nous désignons ici par ce terme est un acte dont les motivations et les finalités peuvent être dépourvues de toute dimension affective. Ceci permet de mieux isoler les mécanismes de création du « capital social ». En sociologie, on emploie cette notion pour désigner l'ensemble de ressources relationnelles qui participent à l'affirmation d'un individu à l'intérieur de son contexte social [19, 20]. Comme le capital économique est l'ensemble des ressources matérielles qui permettent à une entreprise de prospérer sur le marché, dans les réseaux sociaux, le capital social est l'ensemble des relations humaines qui permettent à un individu tions de départ. Le profil 1 affichera donc de plus en plus d'informations sur moi et sur mon corps. Le profil 2 (autrement dit « profil témoin ») affichera la même quantité initiale d'informations. J'arriverai de cette façon à isoler les effets de la présentation de soi sur le capital social.

Pour ce qui est du choix des traces monodimensionnelles de la présence, par exemple les noms d'utilisateur de ces profils, Facebook met en œuvre depuis longtemps une politique d'accès consistant à exiger le «vrai nom » de l'utilisateur. Bien sûr, les exceptions sont nombreuses, mais dans notre cas, il n'était pas question de proposer des noms complètement factices. Et ce, principalement pour ne pas introduire d' emblée un biais : statistiquement, en effet, les utilisateurs anonymes ou ceux qui choisissent un nom fantaisiste sont destinés à avoir moins d'amis. Les noms d'utilisateurs que nous nous autorisons à inscrire initialement sont en réalité des «noms d'usage », des sobriquets derrière lesquels les connaissances peuvent deviner 
l'identité de l'enquêteur sans lire son nom complet (qui sera révélé par la suite). Continuons de citer les notes de l'observation :

Je n'ai pas ajouté d'autres indications sur mon compte : pas d'adresse postale, ni le nom de mon employeur ou celui de mon ancienne école, etc. J'ai complété les deux profils en ajoutant deux photos presque identiques : deux gros plans de moi déguisé en Méphistophélès pour une fête. Une image assez reconnaissable, mais dans laquelle mon visage n'apparaît pas de manière explicite.

Au départ donc, la mise en scène de la présence corporelle dans ces deux profils passe exclusivement par un nom et une photo non réaliste. Étant donné l'impraticabilité de l'anonymat total sur Facebook, ces profils ont néanmoins adopté le degré maximal de dissimulation et de circonspection. Les options de protection de la vie privée ont été réglées au maximum de confidentialité : personne ne pourra consulter les deux profils sauf les amis préalablement accrédités.

Afin de recruter des contacts qui acceptent l'«amitié » de ces deux profils dans cette situation expérimentale, nous nous servirons d'un outil analytique appelé « générateur de noms ». Voilà comment le mettre en place :

Mardi 28 avril 2009. En me basant sur le carnet d'adresses de ma boîte mail, j'ai rédigé une liste générale des noms de toutes mes connaissances. Après, pour chaque nom, j'ai calculé mon degré de proximité émotionnelle à l'aide d'un petit logiciel qui s'appelle IOS. J'ai ensuite trié les noms par degré de familiarité avec eux et pour chacun, j'ai spécifié sexe, âge, nationalité, niveau de scolarité, compétences informatiques et niveau socioéconomique. Enfin, j’ai sélectionné parmi mes connaissances cent noms et je les ai répartis en deux listes comparables, de cinquante individus - une pour chaque profil. J'ai alors envoyé une même « demande d'amitié », mentionnant explicitement mon nom : «Salut [nom de l'utilisateur]. C'est Antonio Casilli. Est-ce qu'un profil comme le mien collerait dans ta liste d'amis?

Les personnes sollicitées réagissent, en ajoutant les deux profils à leurs listes de contacts : au bout d'une semaine, ils ont collectionné respectivement quinze et quatorze amis. Ceux qui n'ont pas donné suite à l'invitation seront relancés pendant quelques semaines. De nouveaux amis seront invités.
Le résultat reste pour autant modeste. Les échanges aussi sont en réalité très peu nombreux dans cette première phase. Ils se limitent à quelques messages d'amis nous félicitant d'avoir rejoint le service. Cette difficulté initiale peut être expliquée par la pauvreté de traces corporelles de ces deux profils. À ce stade, les noms et les photos d'utilisateur sont les seuls renseignements auxquels les contacts Facebook peuvent s'accrocher. Les photos «déguisées » provoquent notamment des prises de position très arrêtées :

Vendredi $1^{\text {er }}$ mai 2009. Jusqu'à aujourd'hui, les retours sur les deux profils sont assez négatifs. Les connaissances de sexe féminin surtout ne se gênent pas pour exprimer leur aversion. Une amie définit le profil 1 comme « effrayant», une autre qualifie la photo du profil 2 de « monstrueuse ». Les réactions masculines se limitent à des commentaires amusés ou tout au plus sarcastiques : «Avec une photo pareille, tu ne peux pas te permettre de commentaires sur la mienne », déclare un ami du profil 1. Un autre dit qu'il attendra que sa fille « soit adulte pour la lui montrer, pour qu'elle ne soit pas traumatisée ».

\section{La pression sociale vers le dévoilement de soi}

Réactions stéréotypées par sexe sans doute, et cependant révélatrices du type de pressions auxquelles un utilisateur de média social est soumis lorsqu'il propose une mise en présence de soi et de son propre corps. Les commentaires épidermiques des amis sont assez importants pour se faire une idée du type de sensibilité et de l'orientation esthétique prédominante dans un groupe. Cela n'est pas spécifique aux services de «réseautage ». Les blogs, par exemple, avec leur concert de voix d'auteurs et de lecteurs confondues, ont érigé les commentaires en préceptes fondamentaux. Facebook hérite des fonctionnalités du blogging ou du microblogging: par les mises à jour de leurs statuts, ses utilisateurs peuvent écrire des textes ou suggérer des liens vers d'autres contenus. Sur les deux profils, nous commençons alors à publier de courts messages en forme de descriptions de gestes, d'actions ou de conditions physiques. Ce sont des traces bidimensionnelles. Bien sûr, chaque trace est simultanément une marque d'investissement de l'utilisateur : il s'agit simultanément de «faire vivre » les profils. Les billets s' affichent ainsi sur les murs de Facebook : 
FIGURE 1.

Graphes de deux profils Facebook

renseignés de manière identique et sommaire

Profil authentique

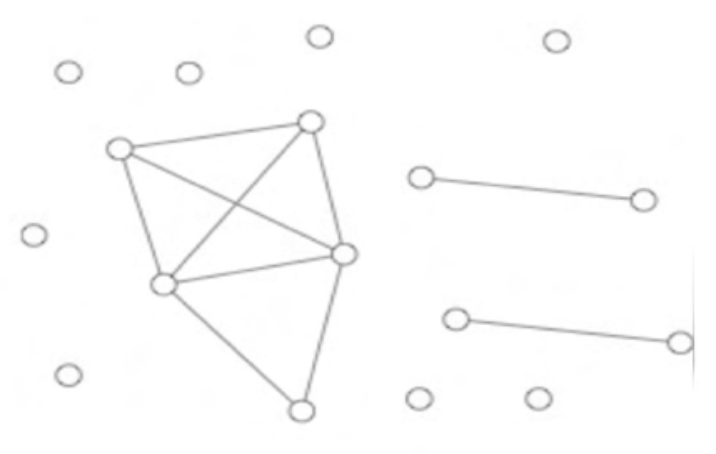

Profil témoin

4 Pour une introduction à nants : il faut plutôt parvenir à créer collectivement une identité faite d'échanges et de capacité à écouter les autres.

Dans les médias sociaux, les utilisateurs qui réussissent le mieux sont ceux qui révèlent le plus d'informations à propos d'eux-mêmes [22]. Le maître mot ici est « dévoilement» : la démarche consistant à s'ouvrir aux autres. Pour vérifier cette hypothèse, il faudra différencier les deux profils, qui ont jusque-là proposé la même présentation. À partir de ce moment-là, le premier profil s'ouvrira progressivement en ajoutant de plus en plus de photos, de textes et davantage de détails significatifs sur ses actions, ses goûts, et même son état de santé. Le «profil témoin », au contraire, continuera à proposer presque la même quantité d'informations, sans se soucier des réactions de ses contacts. Si l'hypothèse de la pression sociale vers le dévoilement est correcte, le premier profil aura un réseau personnel plus riche que le second.

\section{Le graphe social, miroir de la présence en ligne}

Mais comment faire pour savoir si le réseau personnel de chaque profil (le «graphe social» de ses connaissances) devient plus riche? Quels sont les indicateurs structuraux d'un capital social plus important? Il serait superficiel de se limiter à compter le nombre d'usagers qui acceptent la requête d'ajout à leur liste de contacts ou le nombre de commentaires sur les pages d'accueil de mes profils. Une approche sociologique s' est justement spécialisée dans l'analyse des réseaux sociaux ${ }^{4}$. Initialement développée il y a Lundi 4 mai 2009, 23 h 08 - Tony Cas rit à gorge déployée en lisant un article sur arxiv.org [...] ; lundi 11 mai 2009, 11 h 01 - Ant Cas est alité depuis 2 jours [...] ; mardi 12 mai 2009, 08 h 45 -Tony Cas informe qu'il était malade mais assure que ce n'était pas la grippe porcine [...]; mercredi 13 mai 2009, $09 \mathrm{~h} 54$-Ant Cas prend $\mathrm{rdv}$ avec son ophtalmo parce qu'il n'y voit pas clair dans toute cette histoire...

Les « amis » agrémentent ces notes de leurs points de vue et avis discordants - ainsi que d'occasionnels signes d'appréciation, comme l'incontournable pouce levé du «j'aime». Au bout de deux semaines d'expérience, le nombre des contacts n'évolue pas pour autant. Cette réflexion est consignée au carnet d'observations :

Pour mieux me faire accepter, je dois arriver à mieux gérer l'impression de moi que je donne. Mais il ne s'agit pas de produire des récits de soi impression- déjà plus de cinquante ans pour étudier les structures des familles et des organisations, cette approche s'applique aussi parfaitement à l'étude des liaisons numériques. Elle consiste à représenter chaque personne comme un «nœud», un point connecté à d'autres points par des lignes droites («arêtes ») qui représentent des relations.

En l'occurrence, pour chacun des profils Facebook, nous pouvons dessiner un graphe où chaque nœud représentera un membre de la liste de contacts, et chaque arête un lien existant entre eux.

Lundi 11 mai 2009. Aujourd'hui, grâce à un logiciel d'analyse des réseaux sociaux, j'ai obtenu un instantané des réseaux d'amis de mes deux profils. Ils ne sont ni très denses ni très peuplés. À peine 17 membres chacun. Dans la plupart des cas, les nœuds ne sont pas connectés entre eux. 
Je reproduis ici les deux graphes (figure 1).

Ces simples visualisations s'accompagnent du calcul d'indicateurs tels le degré de cohésion ou la densité. Même sans avoir de formation en analyse des réseaux, des structures sociales si réduites peuvent facilement être analysées. On détecte davantage de nœuds isolés dans le profil 1 que dans le profil 2, mais les deux profils sont en réalité comparables : ils comprennent des individus qui connaissent l'utilisateur, mais qui (apparemment) ne se connaissent pas entre eux.

Au centre du premier graphe, on remarque aussi un petit cluster, une agglomération : c' est un groupe d'amis qui sont connectés entre eux sur Facebook et qui, tous, ont accepté de se connecter à l'utilisateur «Tony Cas ». Dans le graphe de l'utilisateur «Ant Cas », par contre, on détecte une triade d'amis (le triangle sur la droite) et quelques dyades (dans trois cas sur quatre, il s'agit de couples mariés ou liés sentimentalement).

La semaine suivante, la situation va changer drastiquement pour le profil 1, à la suite d'une démarche appuyée de dévoilement d'informations personnelles. Entre le 12 et le 19 mai, deux photos sont ajoutées sur ce profil : cette fois-ci, le visage est reconnaissable. Le profil 1 s'investit davantage dans les échanges avec les autres, en laissant plus de commentaires à la première personne. Il commence aussi à révéler ses goûts et préférences en termes de musique, de gastronomie, de livres.

Ces communications sont très pertinentes pour notre propos, qui est et reste la représentation de la présence à travers la monstration du corps en ligne : en indiquant que l'utilisateur du profil 1 apprécie la cuisine japonaise et écoute de la musique punk, en suggérant qu'il lit des bandes dessinées et des poètes de la beat generation, nous proposons une mise en scène très précise de son style personnel. Inconsciemment, on imagine une apparence physique, des vêtements... L'expression des goûts dans la vie de tous les jours participe d'un processus social que l'on qualifie, après Pierre Bourdieu, de «distinction »|25, 26]. Processus complexe, qui consiste à trouver le juste équilibre entre l'envie de se démarquer des autres en affirmant son individualité, et le besoin de se conformer aux goûts dominants de son milieu social. Dans les services de networking en ligne comme Facebook, le même mécanisme est observable. Les pratiques de consommation cultu-
FIGURE 2.

Le profil 1 a affiché ses goûts

Profil authentique

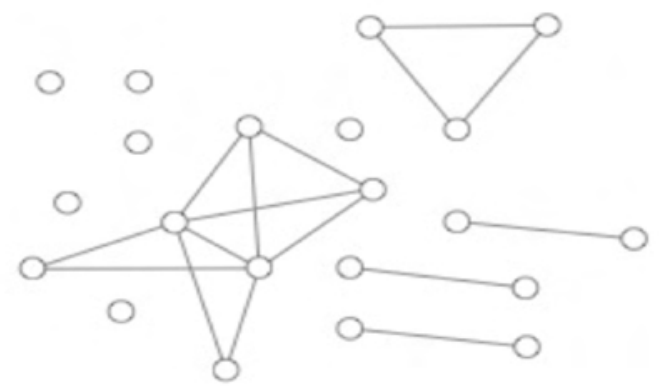

Profil témoin

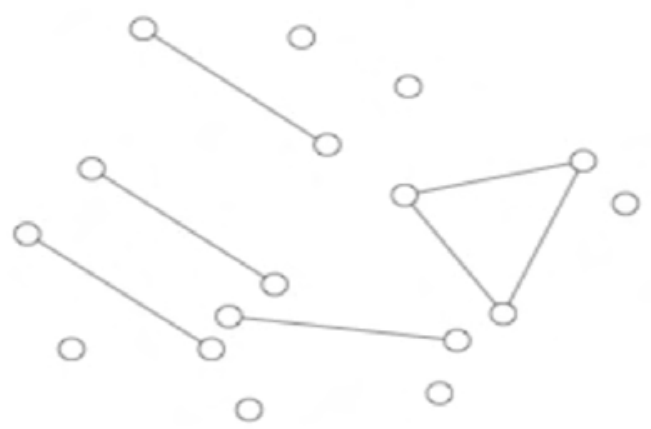

relle (musique, livres, films, etc.) qui s'affichent sur les profils des utilisateurs fournissent certainement des éléments d'ostentation et de théâtralisation de la présence en ligne. Mais si, dans un premier temps, les goûts déclarés par l'utilisateur du profil 1 affirment son identité par rapport aux autres, bientôt l'interface même de Facebook change la donne.

Mardi 19 mai 2009. Le profil 1, qui est constamment ouvert dans mon navigateur, affiche en automatique des petites fenêtres contenant des suggestions ou des «morceaux choisis» par ses amis. «L'utilisatrice $\mathrm{X}$ est fan de l'artiste peintre Tel »; «L'utilisateur Y a aimé le dernier livre de l'écrivain Telautre». Bien sûr, il y a un fond d'agonisme et d'ostentation. Ce sont des façons de proclamer leur «bon goût» face aux autres membres du réseau, de les devancer sur le terrain 
FIGURE 3.

Dans les deux profils tous les champs biographiques ont été renseignés

Profil authentique

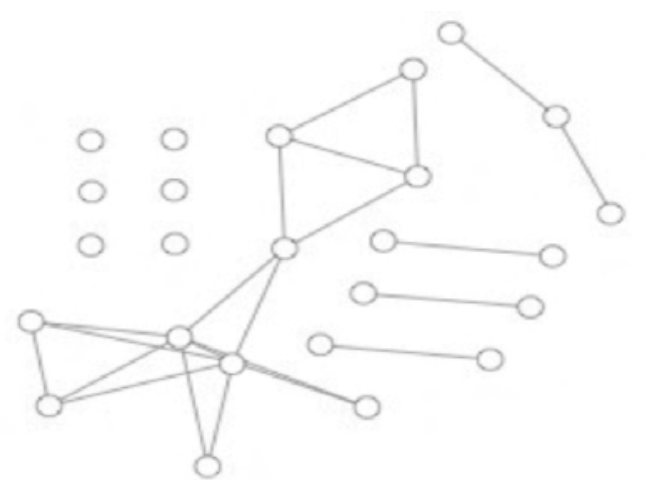

Profil témoin

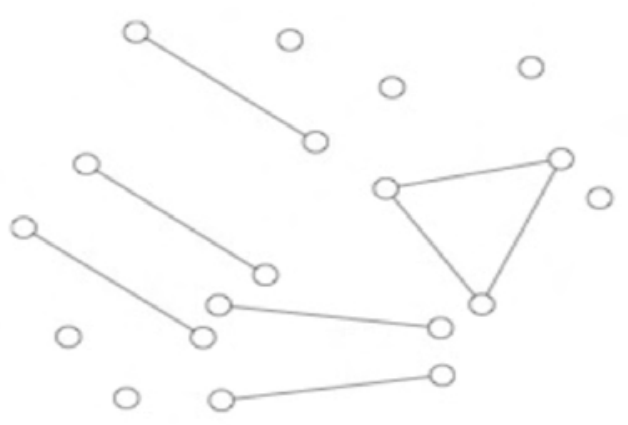

du cool ou du sophistiqué. Mais, au fil des échanges, ce que je voyais au début comme une affirmation de prestige personnel se transforme en une manière de partager avec sa petite communauté de menus et grands plaisirs.

Les goûts et les styles commencent à s'harmoniser quand l'utilisateur du profil 1, à l'instigation de ses amis, devient fan de musiciens « électro » (qu'il n'aimait pas, a priori), ou commence à apprécier les recettes maghrébines. De la mise en présence de soi visant la distinction, on passe à la mise en présence qui sert à tisser des liens sociaux. Liens qui se resserrent, en même temps que le graphe du réseau d'amis du profil 1 se fait plus dense et s'accroît légèrement (21 nouds), suite à l'arrivée d' «amis d'amis » qui demandent maintenant à être ajoutés à sa liste.

Sans aucun doute, la caractéristique la plus remarquable du graphe du profil 1 datant d'après la stratégie de dévoilement (figure 2) est que deux petits clusters de nouds se sont désormais formés - l'un au centre, l'autre en haut à droite. Le premier groupe, qui existait avant, se consolide. Le second est une nouvelle formation. Il s'agit dans tous les cas d'individus qui se fréquentent en dehors de Facebook, de collègues et de camarades unis par des liens forts de proximité personnelle, politique et même économique.

\section{Cohésion ou connectivité?}

Nous ne pouvons donc pas supposer que quelques commentaires en ligne sur les consommations musicales ou gastronomiques du profil 1 ont fait augmenter la cohésion sociale (ce que l'on appelle en anglais «bonding») qui existait déjà entre ses «amis ». Ce que le graphe nous apprend est seulement que, après ces commentaires, ces amis commencent à s'afficher au sein du réseau personnel de l'utilisateur en tant que « cliques ». Par la même occasion, ils invitent l'utilisateur du profil à faire partie de ce groupe homogène.

Au contraire, le profil témoin, qui n'a pas réalisé ce travail d'adaptation stylistique, reste à un nombre constant de membres et aucun nouveau lien ne s'affiche entre ceux-ci. Sa retenue est interprétée comme un désinvestissement, inévitablement. Son réseau d'amis est alors un reflet emblématique de son identité en ligne : raréfié, incohérent, insipide. Face à une mise en scène de soi qui ne se renouvelle pas, les amis expriment leur soupçon et refusent à leur tour de révéler leurs propres affinités avec d'autres membres ou leur appartenance à des groupes. Ces membres de Facebook ont accepté la requête d'ajout à une liste d'amis, bien sûr, mais cela ne suffit pas. S'il n'y a pas de réciprocité dans l'échange de traces de présence, d'informations, de suggestions, d'éléments de narration de soi, les amis ne cautionnent pas l'identité affichée par l'utilisateur du profil témoin.

L'exhibition du corps - de son apparence, de ses gestes et de ses sensibilités - cache aussi un enjeu de confiance réciproque. Mais quel poids attribuer à cet enjeu? Peut-être qu' une validation « officielle » pourrait suppléer à ce défaut de confiance. Au moment de la création des deux profils sur Facebook, les questions relatives à l'âge, au sexe, au lieu de résidence et aux activités des deux utilisateurs ont été délibérément éludées. Ces informations personnelles, requises et cautionnées par une source institutionnelle (le propriétaire même du site), contribuent-elles à 
former l'idée que les amis d'un utilisateur se font de sa présence physique?

Jeudi 21 mai 2009. J'ai spécifié le sexe, la date de naissance, le lieu de résidence et l'employeur pour les deux profils. J'ai surtout remplacé les sobriquets par mon vrai nom. Le profil 1, qui avant s'appelait Tony Cas, s'appelle maintenant Antonio A. Casilli. Le profil 2 (témoin), qui s'appelait Ant Cas, s'appelle Antonio Casilli. Le tout semble maintenant plus transparent, plus en règle avec les conditions générales d'usage du site.

Mais le fait de dévoiler son identité civile entraîne en réalité peu de changements. Le profil témoin gagne un seul membre et une autre dyade se forme, mais pas de nouvelles agglomérations : les amis continuent de ne pas le considérer comme apte à intégrer leurs groupes.

Le profil 1, en revanche, affiche un phénomène intéressant: les deux clusters formés quelques jours auparavant sont à présent reliés par un individu commun (figure 3). Il s' agit de quelqu' un qui, devenu ami des membres des deux composants, joue un rôle de passeur. sert justement à prévenir cette éventualité. Il assure la communication entre les deux agglomérations. Ronald S. Burt, l'une des figures de pointe de l'analyse des réseaux sociaux, dirait que, entre les deux groupes, il y avait un «trou structural» (structural hole), un fossé sur lequel l'ami commun a «jeté un pont» (bridging) [27].

Le capital social peut alors être maximisé à partir du moment où, à l'intérieur d'un réseau, on arrive à trouver le juste équilibre entre cohésion (bonding) au niveau de petits groupes et connectivité (bridging) entre ces mêmes groupes - laquelle est justement assurée par ces individus qui sont à la jonction entre les différents clusters.

Conformément au calendrier initialement établi, la fin de l'expérience approche. Il ne reste qu'à achever la mise en présence du profil 1 par la publication d'un album de photos.

Lundi $1^{\text {er }}$ juin 2009. Ajouté un album dans la section «Mes photos » du profil 1. Aucune image n'a été ajoutée au profil 2. J'ai choisi des instantanés à la qualité médiocre, pris dans des lieux publics entre septembre 2007 et août 2008. Dans ces

\section{"L'exhibition du corps cache aussi un enjeu de confiance réciproque"}

Difficile, pour ce profil, d'affirmer qu'il s'agit d'une conséquence de l'introduction des informations officielles sur ses vrais nom, sexe, âge, etc. Il serait plus correct d'interpréter ce phénomène comme un effet cumulatif de ces nouveaux renseignements et des informations sur ses goûts livrées la semaine précédente. Toujours est-il que cette jonction entre les deux groupes est un signal de l'enrichissement du réseau personnel du profil 1. La richesse d'un réseau n'est pas seulement représentée par sa taille et par le nombre de nœuds. Même si les membres sont désormais vingtcinq, cela ne servirait à rien s' ils n' avaient un bon niveau de cohésion entre eux. Cette cohésion existe depuis que deux clusters homogènes d'amis se sont formés.

Mais encore, cela ne suffirait pas pour faire un «bon» réseau d'amitiés. Les groupes pourraient entrer en conflit et, finalement, détruire sa cohérence interne. L'apparition de cet individu «passerelle » photos, je ne pose pas. Cet élément est important : les portraits sont censés être des images «spontanées ». Par ailleurs, dans les photos, je ne suis pas en compagnie d'autres personnes, pour éviter que leur apparence introduise un biais dans la réception de ma propre mise en scène de moi.

Apparemment, le manque d'artifice n'est pas apprécié par les amis du profil 1. En privé (messages personnels) et en public (messages pouvant être vus par d'autres utilisateurs), les commentaires soulignent la mauvaise qualité des images, ou même le jour peu favorable sous lequel certaines montrent le sujet. («T'en avais pas de meilleures?», demande une amie portugaise ; un ami italien surenchérit : «Qu'est-ce qu'il lui est arrivé à l'auteur de cette photo? Ils l'ont fusillé sur la place Rouge, après ?»).

Et pourtant, ces commentaires négatifs ne se traduisent pas en une diminution du capital social. 
FIGURE 4.

Un album photo à été mis en ligne sur le profil 1

Profil authentique

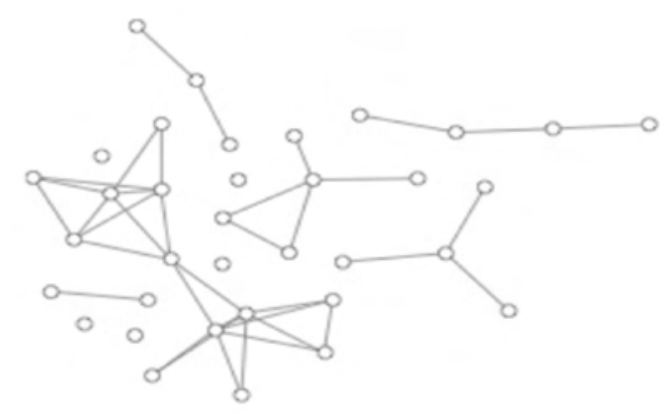

Profil témoin

Le réseau d'amis de ce profil continue de s'accroître en taille, et les connexions entre ses membres se développent encore. Il pourrait toutefois s'agir d'une coïncidence fortuite. Une dernière démarche permet de vérifier ce point : le 11 juin, une invitation explicite est remise aux « amis » du profil 1 pour qu'ils regardent l'album de photos. Dans les jours qui suivent, encore plus de commentaires critiques. Mais, paradoxalement, encore plus de liens se créent entre les membres du réseau d'amis du profil 1, lequel affiche désormais quatre clusters, dont deux reliés entre eux. Des trentecinq membres qui le constituent désormais, à peine $14 \%$ ne sont pas connectés entre eux (contre $47 \%$ initialement). Le profil 2, comme il était prévisible, n'a pas évolué, exception faite d'un membre isolé qui est venu s'y ajouter.

\section{Individu et communauté : une co-évolution}

Le sens de cette expérience est que, quand il s'agit de maximiser son capital social dans un site de networking comme Facebook, la mise en présence de soi et de son apparence est moins une activité individuelle que groupale. Il ne s'agit plus d'exprimer ce que l'on est ou ce que l'on veut être. La présence ne jaillit pas d'une narration individuelle, mais surgit du partage et de la conversation avec les autres. C'est pourquoi même une présence corporelle perçue comme imparfaite (c'est le cas des photos trop «spontanées ») n'affecte pas négativement la qualité du réseau dans la mesure où elle devient occasion d'échanges et de construction d'une identité collective [28]. Cette expérience nous aide à encadrer formellement le rôle des liaisons numériques, attesté autant par la croissance du graphe social que par les commentaires d' «amis ». La communauté des usagers est, finalement, l'arbitre ultime du succès ou de l'échec d'une présentation de soi en ligne. Ce que l'on peut se permettre de montrer de soi - au-delà des contrôles des entreprises propriétaires des plateformes sociales, et au-delà même des envies de s'afficher sous un jour nouveau par rapport à ce que l'on est au quotidien - est au banc d'épreuve des communautés d'usagers.

Ceci ne veut pas pour autant dire que le réseau écrase systématiquement les individualités. Au contraire, la tendance que l'on appelle en sociologie l' « homophilie » (la disposition à s'associer de préférence avec des personnes qui nous ressemblent), n'est pas prépondérante. Dans la formation des réseaux en ligne, les liens affinitaires ne seraient pas exclusivement créés en fonction de similarités préexistantes. Les utilisateurs peuvent aussi poursuivre des stratégies de personnalisation et de mise en présence visant à faire apparaître des spécificités individuelles parfois marquées qui les distinguent des autres sans pour autant les en aliéner. En accentuant les variations intra-individuelles, ces spécificités peuvent être favorables à la formation de liens de type bridging (connectivité sociale), sans exclure complètement le bonding (cohésion sociale), dans la mesure où ces variations sont socialement acceptées.

En décrivant comment la validation collective de la présence, des styles et des postures individuelles s'articule avec la formation de réseaux personnels dans un contexte d'interactions sociales sur 
Internet, nous avons décrit une double dynamique. D'une part, la présentation de soi a un impact sur la création d'un réseau personnel et son évolution progressive. De l'autre, la constitution du réseau personnel d'un individu et ses changements dans le temps finissent à leur tour par influer sur les straté- gies de présence, sur les caractéristiques physiques et culturelles affichées dans les profils. On peut qualifier cette double influence de « co-évolution »: la structure des réseaux s'adapte aux membres et les membres s'adaptent aux changements de la structure.

\section{Bibliographie}

[1] CAsıLı A. A., « Les avatars bleus : autour de trois stratégies d'emprunt culturel au cœur de la cyberculture », Communications, $n^{\circ} 77$,

2005, p. 183-209.

[2] Coulmont B., Sociologie des prénoms, Paris, La Découverte, coll. « Repères », 2011.

[3] Back M. D., Schmuke S. C., Egloff B., « How Extraverted is honey.bunny77@hotmail.de? Inferring Personality from e-mail Addresses », Journal of Research in Personality, vol. 42, $n^{\circ}$ 4, 2008, p. 1116-1122.

[4] GuntherT A., « "Sans retouche" : Histoire d'un mythe photographique », Études photographiques, vol. XXII, n 1, 2008,

http://etudesphotographiques.revues.org/index1004.html [dernier accès 24 juin 2012].

[5] Goffman E., The Presentation of Self in Everyday Life, Garden City, N.Y., Doubleday, 1959.

[6] Velkovska J., Beaudouin V., « Constitution d'un espace de communication sur Internet (forums, pages personnelles, courrier électronique...) », Réseaux, n 97, 1999, p. 131.

[7] Casilu A. A., Tubaro P., Araya P., « Ten Years of Ana: Lessons from a Transdisciplinary Body of Literature on Online pro-eating Disorder Websites », Social Science Information, vol. LI, n 1, 2012, p. 121-139.

[8] Strategy Analytics, « Virtual Worlds Projected to Mushroom to Nearly One Billion Users », Communiqué de presse du 28 mai 2008, consultable sur www.strategyanalytics.com. [dernier accès 24 juin 2012].

[9] Cooper R., Alter Ego. Avatars and Their Creators, Londres, Chris Boot, 2007.

[10] Bergström M., «Sites de rencontres : nouveaux territoires sexuels, nouvelles frontières », Poli-Politique de l'image, n 4, 2011, p. 99-111.

[11] Martin L. H., Gutman H., Hutton P. H. (DIR.), Technologies of the Self: A Seminar with Michel Foucault, Amherst, University of Massachusetts Press, 1988.

[12] Allard L., VanderberGhe F., «Express Yourself! Les pages perso. Entre légitimation techno-politique de l'individualisme expressif et authenticité réflexive peer-to-peer », Réseaux, n 117, 2003, p. 191-220.

[13] Cardon D., Delaunay-Téterel H., « La production de soi comme technique relationnelle. Un essai de typologie des blogs par leurs publics ", Réseaux, n 138, 2006, p. 15-71.

[14] Utz S., « Show me your friends and I will tell you what type of person you are : How one's profile, number of friends, and type of friends influence impression formation on social network sites », Journal of Computer-Mediated Communication, vol. 15, n 2, 2010, p. 314-335.

[15] Golder S., YARDI S., « Structural Predictors of Tie Formation in Twitter : Transitivity and Mutuality », Proceedings of the Second IEEE International Conference on Social Computing, August 20-22, Minneapolis, MN, 2010.

[16] Zimmer M., « Subject Privacy and the Release of the Tastes, Ties, and Time Dataset », Workshop on Revisiting Research Ethics in the Facebook Era: Challenges in Emerging CSCW Research, Computer Supported Cooperative Work Conference, Savannah, GA, 2010.

[17] Casıuı A. A., « Mon friend n'est pas mon ami », in Les Liaisons numériques. Vers une nouvelle sociabilité ?, Paris, Seuil, 2010.

[18] Douelн! M., Pour un humanisme numérique, Paris, Seuil, 2011.

[19] Bourdeu P., « Le capital social. Notes provisoires ", Actes de la recherche en sciences sociales, n 31, 1980, p. 2-3. Voir la rubrique « [Re]découvertes » de ce numéro.

[20] Coleman J. S., « Social Capital in the Creation of Human Capital », American Journal of Sociology, Supplement to vol. LXXXIV, 1988, p. 95-120.

[21] LiN N., « Building a Network Theory of Social Capital », Connections, vol. XXII, n 1, 1999, p. $28-51$.

[22] Trammel K. D., Keshelashvili A., « Examining the New Influencers: A Self-presentation Study of A-List Blogs », Journalism and Mass Communication Quarterly, vol. LXXXII, $n^{\circ} 4,2005$, p. 968-982.

[23] MerckLÉ P., Sociologie des réseaux sociaux, Paris, La Découverte, coll. « Repères », 2011.

[24] Lazega E., Réseaux sociaux et structures relationnelles, Paris, PUF, coll. « Que sais-je ? », 2007.

[25] Bourdieu P., La Distinction. Critique sociale du jugement, Paris, Minuit, 1979.

[26] Lahire B., La Culture des individus. Dissonances culturelles et distinction de soi, Paris, La Découverte, 2004.

[27] Burt R. S., Brokerage and Closure. An Introduction to Social Capital, Oxford, Oxford University Press, 2005.

[28] Zhao S., Grasmuck S., Martin J., « Identity Construction on Facebook : Digital Empowerment in Anchored Relationships », Computers in Human Behavior, vol. XXIV, n 5, 2008, p. 1816-1836. 